\title{
Optimal PMUs Placement Due to Several Stages for Achieving Full System Observability
}

\author{
Mohamed A. Badr, Ahmed. A. Hossam-Eldin and Nabil. H. Abbasy \\ Electrical Engineering Department, Alexandria University, Alexandria, Egypt
}

\begin{abstract}
The new measurement devices such as phasor measurement units (PMUs), is considered to be an essential component in implementing future power network systems. The problem of optimal placement of PMUs (OPP) requires finding a minimum numbers of PMUs that must be installed to provide full system observability. Due to the large number of the PMUs required and their relatively high cost, it is important to partition the installation of PMUs placement into several stages to overcome this problem. A new proposal depends on limiting the number of installed PMUs at each stage and maximizing system observability will be illustrated in this paper. The proposed method will be implemented on IEEE-14 bus system and IEEE-30 bus system.
\end{abstract}

\section{INTRODUCTION}

Operation of the power system is becoming very complicated and facing many challenges every time due to the expanding of the power generation and transmission utilities. The power systems have also become so interdependent that the events in one area can cascade and have significant impact on other remote areas. This change in the industry is putting pressure for the development of new tools to monitor system stability and reliability.

Until recently, available measurement sets did not contain phase angle measurements due to the technical difficulties associated with the synchronization of measurements at remote locations. Global positioning satellite (GPS) technology alleviated these difficulties and lead to the development of phasor measurement units (PMUs).

Synchronized Phase Measurement Unit (PMU) is a monitoring device, which was first introduced in mid1980s. Phasor measurement units (PMU) are devices, which use synchronization signals from the global positioning system (GPS) satellites and provide the phasors of voltage and currents measured at a given substation. As the PMUs become more and more affordable, their utilization will increase not only for substation applications but also at the control centers for the EMS applications [1].

All Synchrophasor measurements are time tagged with the Coordinated Universal Time, via GPS. All GPS receivers generate a very accurate pulse, which is used by PMUs to generate very precise sampling pulses. PMUs provide a more exact view of the system because samples are taken and time stamped in a microsecond sampling window and although data from multiple units can only be available and time aligned in the order of milliseconds the time stamp included with the measurements eliminates the errors introduced in the state estimator by the size of the scanning window.

Different methods which are used to compute the minimum required numbers of PMUs for full system observability will be described in part II.

Importance of PMU in wide area monitoring system, and PMUs placement based on system decomposition will be illustrated in part III.

Different methods which are used for multi-stage of PMUs placement in power system will be described in part IV. Implementation of optimal multi-stage PMUs placement will be carried out on IEEE-14 bus and IEEE-30 bus systems, part V.

\section{OPTIMAL PMUS PLACEMENT FOR FULL POWER SYSTEM OBSERVABILITY}

The main objective from this section is to explain different methods which are used for optimal PMUs placement to make the system fully observable. An integer linear programming (ILP) approach is used to determine the optimal number and locations of PMUs. PMUs placement guarantees the system observability during normal operating conditions as well as single branch outages or single PMU outages. The proposed scheme is applied to the IEEE-14 bus and IEEE-30 bus systems. 


\section{A. OBSERVABILITY RULES FOR PMUS PLACEMENT}

A PMU is able to measure the voltage phasor of the installed bus and the current phasors of some or all the lines connected to that bus. The following rules can be used for PMUs placement:

Rule 1; assign one voltage measurement to a bus where a PMU is placed, including one current measurement to each branch connected to the bus itself.

Rule 2; assign one voltage pseudo-measurement to each node Reached by another equipped with a PMU.

Rule 3; assign one current pseudo-measurement to each branch connecting two buses where voltages are known. This allows interconnecting observed zones.

Rule 4; assign one current pseudo-measurement to each Branch where current can be indirectly calculated by the Kirchhoff current law (KCL). This rule applies when the current balance at a node is known [2].

\section{B. INTEGER LINEAR PROGRAMMING(ILP) FOR PMUS}

A PMU placed at bus i will measure the voltage phasor of bus $i$ and a predetermined number of phasor currents of outgoing branches of that bus. The number of the measured current phasors depends on the number of PMU channels made available.

It is assumed that a PMU placed at bus $\mathrm{i}$ will measure all current phasors of the branches connected to that bus, in addition to the voltage phasor of bus $\mathrm{i}$. Therefore, with the absence of any conventional measurements in the system, bus i will be observable if at least one PMU is placed within the set formed by bus $i$ and all buses incident to it. The zero injection bus, single PMU outage and transmission line failure will be considered.

Therefore, the objective of optimal PMU placement problem is to determine the minimum number of PMUs so as to preserve the system Observability [3]. This objective can be formulated as:

$$
\min \sum_{i \in I} x i
$$

$$
\mathrm{fi}=\sum_{j \in I} a_{i j} x^{i} \quad \forall i \in I
$$

$$
f i \geq 1
$$

$\forall i \in I$

$$
a i j=\left\{\begin{array}{l}
1 \\
1 \\
0
\end{array}\right.
$$

\section{if $i=j$
ifbusesiandjareconnected otherwise}

Where:

$\mathrm{Xi} \quad$ is a binary decision variable associated with bus $\mathrm{i}$

I is the set of buses;

$\mathrm{fi} \quad$ is the observability function related to bus $\mathrm{i}$;

aij is a binary decision variable associated with

bus $i$ and $j$

Table 1. Results of OPP for IEEE-14 bus system

\begin{tabular}{|c|c|c|}
\hline Scenario & $\begin{array}{c}\text { Min No of } \\
\text { PMUs }\end{array}$ & Optimal locations \\
\hline Normal topology & 4 & $2,6,7,9$ \\
\hline Modeling of ZI & 3 & $2,6,9$ \\
\hline $\begin{array}{l}\text { Single PMU outage } \\
\text { with modeling of ZI }\end{array}$ & 7 & $2,6,9,4,5,10,13$ \\
\hline
\end{tabular}

\section{PMUS PLACEMENT BASED ON SYSTEM DECOMPOSITION [4]}

Due to the large required number from PMUs to fulfill power system observability it is considered economically infeasible to install all these PMUs in one shot. The optimal PMUs placement (OPP) is divided into muti-stage installation. This means solving the OPP in incremental placement strategy. Area system decomposition by using the spanning tree method will be illustrated. The optimal number of PMUs for each area will be determined taking into consideration the effect of the internal and boundary buses, using these properties to minimize the total number of PMUs for full power system observability. In this section an approach used for finding the initial partitioning between the buses of the power system will be overviewed. This approach was firstly proposed by Branes [5]. The approach partition the network into many blocks $(m)$ or sub-networks then, it creates a formulation for that partitioning by using 
ILP. The main principle for this approach is to find the minimum spanning tree for the network after that, the system is partitioned using equi-partitioning, multipartitioning algorithm [6] and Kruskal algorithm [7]. Consider a power system with $\mathrm{n}$ buses and partitions. Partitions are separated by tie-lines whose terminal buses are assigned to both the connecting blocks. Thus, after decomposition, each bus will belong to anyone of these two categories:

- Internal bus (XI), if its neighbors belong to block-i. (The bus inside area and is not connected to buses of any other areas.)

- Boundary bus $(\mathrm{Xb})$, if its neighbors are internal buses of block-i and at least one boundary bus from another block. (The bus inside area but connected to buses of external area)

Based on these two categories, six bus vectors are defined as follow:

- Xil - set of internal buses of block-i.

- Xib - set of boundary buses of block-i.

- XiTb - set of total boundary buses (including buses of blocks adjacent to block-i ) associated with block-i.

- $X=\{X i l ; X i b\}-$ set of total buses in block-i.

- $\mathrm{XT}=\{\mathrm{Xil} ; \mathrm{XiTb}\}-$ set of buses of block-i including neighboring boundary buses also.

- $X B$ - set of boundary buses to be excluded from block-i its constraint function.

The optimal PMU placement has been formulated as an Integer Linear Programming problem in order to minimize the total cost of PMU installation and the constraints are formulated using phasor measurements from PMUs along with the pseudomeasurements. Pseudo-measurements are obtained using Ohm's and Kirchhoff's laws. [8].

The following steps have been used to optimally place the PMUs in the sub-networks.

1. Sort the blocks in descending order of their size

2. Start block counter, $i=1$.

3. Identify all set of buses for the block-i i.e. Xil, Xib, XiTb, $X=\{X i l ; X i b\}, X T=\{X i l ; X i T b\}$.

4. Formulate ILP problem for block-i as,
Subject to

$$
\begin{aligned}
& f_{j}\left(x_{i I}, x_{i T b}, x_{B}\right) \geq 1 \\
& \mathrm{x}_{\mathrm{j}}=(0 \text { or } 1) \text { bir }
\end{aligned}
$$

where

- $\quad \mathrm{Cj}$ is the cost of PMU installation at bus. Assume that, cost of installation of each PMU has assumed to be equal and considered as 1 p.u. Thus, in (1) $\mathrm{Cj}=1$.

- $\quad f$ is constraint set; constraint at any bus $-j$ is formulated in such a way that it becomes observable either through direct phasor measurement or phasor pseudo-measurement. This is achieved either by directly placing the PMU at the bus-j or at any one of the buses directly connected to the bus-j.

- $\quad X B$ is the set of boundary buses to be excluded while formulating constraint set of block. This is an essential step; otherwise, each boundary bus would be considered twice for the constraint formulation and would lead to increased optimal PMU solution. Thus, when a boundary bus is once considered for the constraint formulation, it should be added in the XB set so that it cannot be considered again. Clearly, for $i=1$ the first block, $X B$ would be a null vector and after each iteration it has to be updated.

5. Update $X B=\{X B ; X i b\}$.

6. If $i=k$, then Stop, else $i=i+1$ and go to step 3 .

The results of the portioning of IEEE-14 bus system are shown in figure (1)

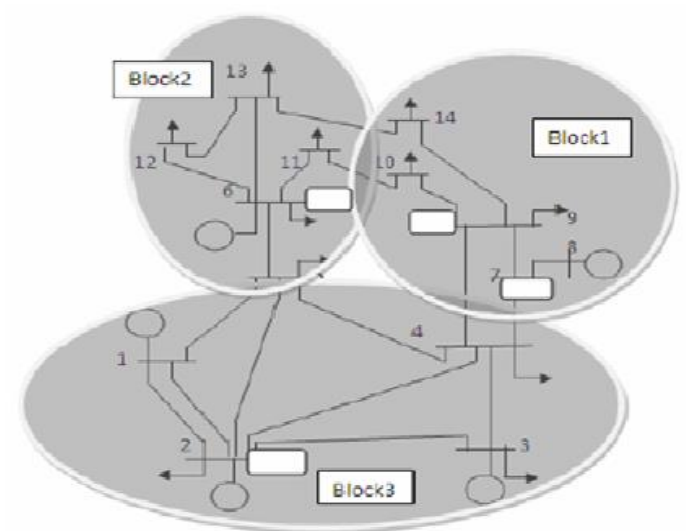

Fig .1. OPP result for IEEE14 bus system after system decomposition [4] 
Table 2. optimal PMU placement results for IEEE-14 bus system

\begin{tabular}{|c|c|c|c|c|}
\hline System & $\begin{array}{c}\text { Number } \\
\text { of } \\
\text { partitions }\end{array}$ & $\begin{array}{c}\text { Number } \\
\text { of buses }\end{array}$ & $\begin{array}{c}\text { Number of } \\
\text { zero } \\
\text { injection }\end{array}$ & $\begin{array}{c}\text { Optimal } \\
\text { PMUs }\end{array}$ \\
\hline IEEE 14 & 3 & $5,5,4$ & 0 & 4 \\
\hline
\end{tabular}

\section{PROPOSED METHOD FOR OPTIMAL MULTI-STAGE OF PMUS PLACEMENT FOR FULL SYSTEM OBSERVABILITY}

In this section the main principle of the proposed algorithm will be explained. The simulation results will be illustrated. The proposed utilizing model depends on maximizing the system observability taking into consideration installing limited number of PMUs at each stage as constraints. The model will be implemented on IEEE-14 bus and IEEE-30 bus systems.

The main principle of the present algorithm is maximizing the observability of the power system subject to a limited number of PMUs at each stage.

By this way a direct relationship between the PMUs placement at each stage and the cost or the available budget during that stage will be considered. The main objective function is to maximize the observability.

Max O

Subject to

No $P M U \leq M$

Where $M$ is the number of PMUs at each stage determined based on allocated budget for the particular stage.

The steps of the proposed algorithm are illustrated as follows:

1. Determine the minimum number of PMUs required for full system observability $\mathrm{N}$, taking into consideration the zero injection buses and the probability of PMU loss or transmission line failure.

2. Determine the number of combinations of choosing M PMUs in our system out of $\mathrm{N}$.

$$
C_{M}^{N}=\frac{N !}{M !(N-M) !}
$$

3. Determine observability index for each combination included in $\mathrm{M}$.
4. Select the particular combination of $M$, which will achieve maximum system observability, and install the M PMUs at those buses at first stage.

5. At the second stage selected $M$ buses at the previous stage will be eliminated.

6. Calculate the number of combinations of choosing $M$ buses from the remaining possibilities or combinations out of (N-M).

7. Determine the observability index for each combination, choose the combination which will achieve maximum Observability for the system and install PMUs at those buses at second stage.

8. If there are many combinations achieve the same observability for the system, the one which achieves maximum number of new observable buses which are not observable at the previous stages will be considered where the remaining buses are neglected.

9. At the next stages the three above steps (5-6-7) will be repeated.

10. If all $\mathrm{N}$ buses have been taken at previous stages, then all PMUs have been installed at the system.

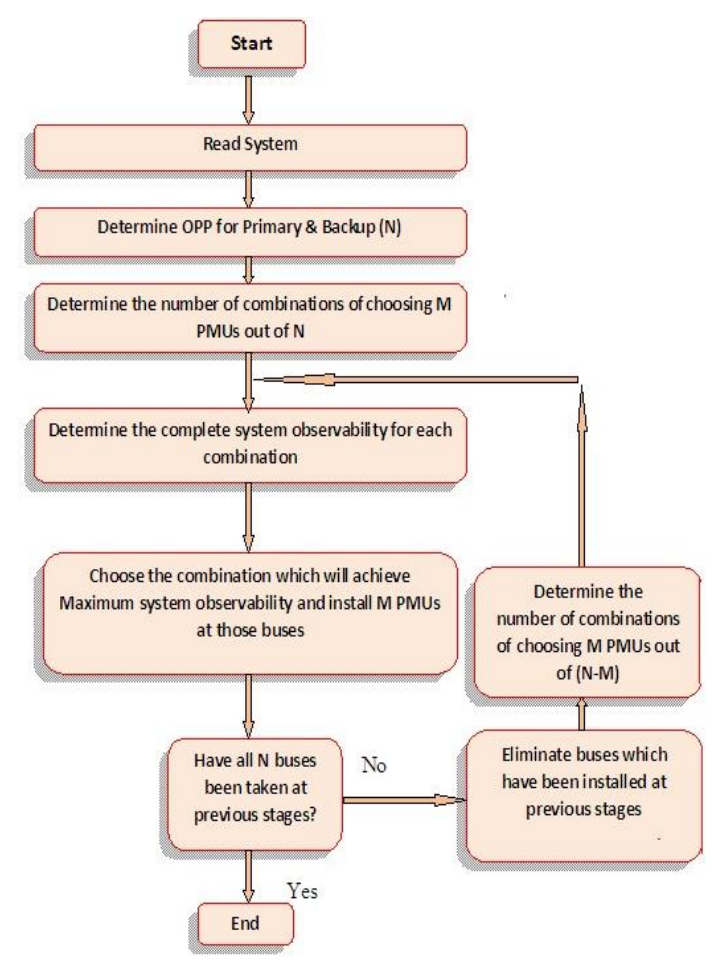

Fig .2. Flowchart of proposed algorithm for carrying out multistage of PMUs placement 


\section{CASE STUDY}

In this study the above steps will be implemented. For simplifying it can be assumed that the number of PMUs which will be installed at each stage $M=2$. The implementation will be carried out for the IEEE-14 bus system and IEEE-30 bus system.

\section{A. IEEE-14 bus system}

From section II, the minimum number of PMUs placement for full power system observability (OPP) of IEEE-14 bus system is seven PMUs as follow:

The OPP $=\left[\begin{array}{lllllll}2 & 6 & 9 & 4 & 5 & 10 & 13\end{array}\right]$

By implementing the above steps in section IV on IEEE-14 bus system, the results are shown in table (3). The combination numbers of choosing 2 PMUs from 7 are 21 as shown in the below equation.

$c_{2}^{7}=\frac{7 !}{2 ! 5 !}=21$

From the simulation results, it is found that, there are three combinations that will achieve same system observability, ten buses will be observable at each one. These combinations are $(6,9),(6,4),(4,13)$. To define which one must be installed at the first stage from the above three Combinations, the maximum number of new observable buses at the second stage which are not observable at first stage for each combination must be defined. After that start with the combination number which achieve maximum number of new observable buses at second stage. from the simulation results it can be concluded that, all three combinations will achieve three new observable buses at second stage. the combination of $(6,9)$ will achieve these three buses four times while the other two combinations will achieve these three buses two times only.

So at first stage it is recommended to install two PMUs at buses $(6,9)$. After the first stage the following buses are observable $\left[\begin{array}{lllll}4 & 5 & 11 & 12 & 13\end{array}\right.$ $\left.\begin{array}{lllll}7 & 10 & 14 & 6 & 9\end{array}\right]$. After completing that stage the system observability will be 0.7143 , as shown in table (3).

At second stage, to define the location of the PMUs which will be installed at the second stage, we should carry out the followings:
Table 3. OMPP for IEEE-14 bus system results

\begin{tabular}{|c|c|c|c|c|}
\hline Stages & 1 & 2 & 3 & 4 \\
\hline Location of PMUs & 6,9 & 2,13 & 4,5 & 10 \\
\hline $\begin{array}{c}\text { Number of } \\
\text { observable buses } \\
\text { at system }\end{array}$ & 10 & 13 & 13 & 13 \\
\hline $\begin{array}{c}\text { New observable } \\
\text { buses }\end{array}$ & Non & 123 & Non & Non \\
\hline $\begin{array}{c}\text { Observable buses } \\
\text { status }\end{array}$ & 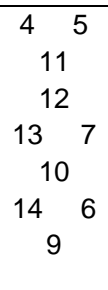 & 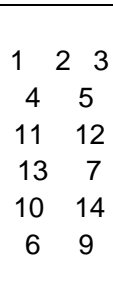 & 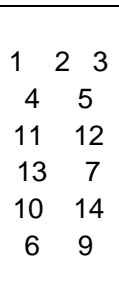 & \begin{tabular}{cc}
1 & 2 \\
3 & 4 \\
5 & 11 \\
12 & 13 \\
7 & 10 \\
14 & 6 \\
\multicolumn{2}{c}{9}
\end{tabular} \\
\hline $\begin{array}{l}\text { Observability } \\
\text { Function }\end{array}$ & 0.7143 & 0.9285 & 0.9285 & 0.9285 \\
\hline
\end{tabular}

\section{Observability}

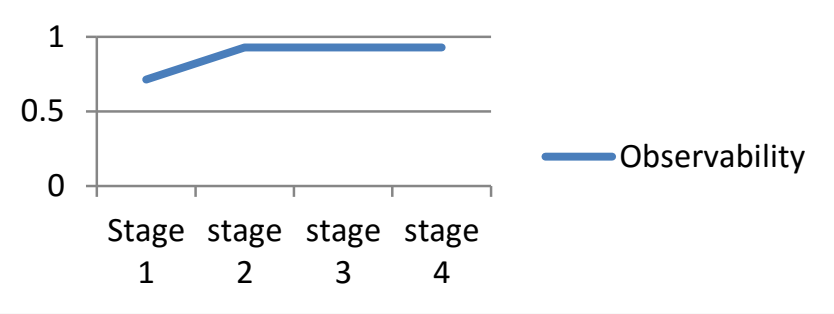

Fig .3. Observability function toward multi-stages of PMUs.

\section{B. IEEE-30 bus system}

The minimum numbers of PMUs placement for full power system observability are 16 PMUs. The optimal PMUs placement is (OPP)

$\mathrm{OPP}=\left[\begin{array}{llllllllll}1 & 3 & 5 & 7 & 9 & 10 & 12 & 13 & 15 & 17\end{array}\right.$ $\left.\begin{array}{llllll}19 & 20 & 22 & 24 & 27 & 29\end{array}\right]$.

The combinations or possibilities of choosing 2 buses from 16 are 120 as follow:

$C_{2}^{16}=\frac{16 !}{2 ! 14 !}=120$

From the results found that, there are one combination $(10,12)$, will achieve maximum system observability, ten buses buses. So at the first stage two PMUs will be installed at buses $(10,12)$. After that stage the observable buses are $\left[\begin{array}{lllll}4 & 6 & 9 & 17 & 20\end{array}\right.$ $\begin{array}{llllllll}21 & 22 & 13 & 14 & 15 & 16 & 10 & 12] .\end{array}$

To define the location of the PMUs which will be installed at the second stage, the following steps must be carried out: 
1. Eliminate any combination which contains bus 10 or bus 12 .

2. Determine the remaining possibilities or combinations of choosing 2 from 14 as follow:

$$
\mathrm{C}_{2}^{14}=\frac{14 !}{2 ! 12 !}=91
$$

For each combination, we should determine the new observable buses which are not observable in the first stage. From the results it can be concluded that, there are two combinations will achieve the maximum number of new observable buses ( 8 buses), which are not observable at first stage these combination are (1, 27) \& $(5,27)$. To define which one must be installed at the second stage, the number of buses which are not observable at the second stage for each one must be calculated. The combination which will achieve the maximum number of new observable buses will be taken. From the results it can be concluded that, it is no difference between the two combination $(1,27)$ \& $(5,27)$, as if two PMUs are installed at buses $(1,27)$ or at $(5,27)$, four new buses will be observable at the third stage which are not observable at the second stage. So at the second stage two PMUs can be installed at buses $(1,27)$ or $(5,27)$. It can be assumed that, at the second stage two PMUs will be installed at buses $(1,27)$.

At the next stages same concept will be implemented. The final results are shown in table (4).

Table 4. Conclusion results of proposed method for OMPP for IEEE-30 bus system

\begin{tabular}{|c|c|c|c|c|c|}
\hline $\begin{array}{l}\text { Stage } \\
\text { uu nuber }\end{array}$ & $\begin{array}{l}\text { FMrts } \\
\text { lecaltou }\end{array}$ & $\begin{array}{l}\text { Netw observ able } \\
\text { brses wh bich are } \\
\text { not observatie at } \\
\text { pretisus stage }\end{array}$ & $\begin{array}{l}\text { Observable buses at the } \\
\text { aytrem }\end{array}$ & $\begin{array}{l}\text { Number of } \\
\text { Osservalise } \\
\text { buses }\end{array}$ & $\begin{array}{l}\text { System } \\
\text { Observ ailitity } \\
\text { fuxction }\end{array}$ \\
\hline 1 & 10.12 & None & $\begin{array}{l}4,6,9,17,20,21,22 \\
13,14,15,15,10,12\end{array}$ & 13 & 0.433 \\
\hline 2 & 1,27 & $\begin{array}{c}2,3,25,28,30, \\
29,1,271\end{array}$ & $\begin{array}{c}4,6,9,17,20,21,22, \\
13,14,15,16,10,12, \\
2,3,25,23,30,29,1, \\
27\end{array}$ & 21 & 0.705 \\
\hline 3 & 1922 & $13,19,24$ & $\begin{array}{c}4,6,9,17,20,21,22, \\
13,24,15,16,10,12, \\
2,3,25,23,30,29,1, \\
27,18,19,24\end{array}$ & 24 & 0.805 \\
\hline 4 & 79 & $5,: 1,7$ & $\begin{array}{l}4,6,9,17,20,21,22, \\
13,14,15,16,10,12, \\
2.3 .25,23,30.29,1, \\
27,18,19,24,5,11,\end{array}$ & 27 & 0.905 \\
\hline 5 & 3,15 & 23 & $\begin{array}{c}4,6,9,17,20,21,22, \\
13,14,15,16,10,12, \\
2,3,25,23,30,29,1, \\
27,13,19,24,5,11,7, \\
23\end{array}$ & 28 & 0.933 \\
\hline 6 & 5,13 & None & $\begin{array}{c}4,6,9,17,20,21,22, \\
13,24,15,16,10,12, \\
2,3,25,23,30,29,1, \\
27,13,19,24,5,11,7, \\
23\end{array}$ & 28 & 0.933 \\
\hline 7 & 17,20 & None & $\begin{array}{c}4,6,9,17,20,11,22, \\
13,24,15,16,20,12, \\
2,3,25,23,30,29,1, \\
27,13,19,24,5,11,7, \\
23\end{array}$ & 28 & 0.933 \\
\hline 8 & 24,29 & None & $\begin{array}{c}4,6,9,17,20,21,22, \\
13,14,15,16,10,12, \\
2,3,25,23,30,29,1, \\
27,13,19,24,5,11,7, \\
23\end{array}$ & 28 & 0.933 \\
\hline
\end{tabular}

From the above simulation results on the two systems it can be concluded that, the complete system observability is achieved much before the last stage of installation of PMUs. Also all the tie lines become observed well before the last stage of PMUs placement. Since the PMUs placement is robust against a PMU failure or single line outage.

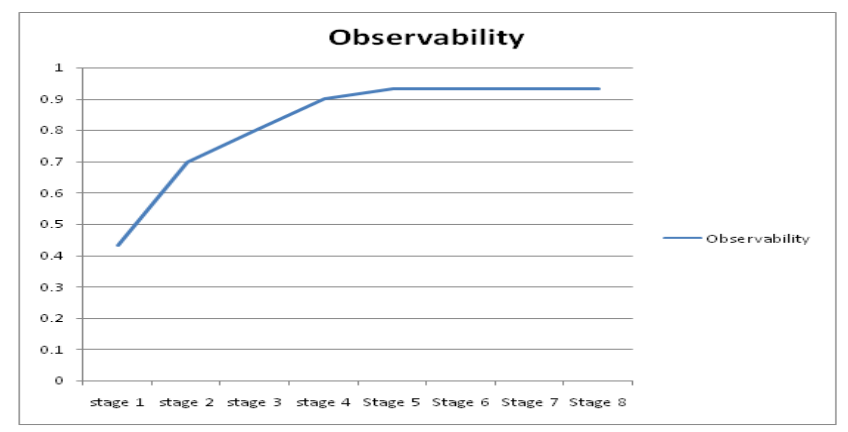

Fig .4. Observability function belongs to number of stages for IEEE-30 bus system.

\section{CONCLUSION}

Improvements in power system control and protection is achieved by utilizing real time synchronized phasor measurements PMUs. The trend in recent years is the steady increase of Phasor Measurement Units (PMUs) installations worldwide for various applications. Placement of phasor measurement units (PMUs) in power systems has often been formulated for achieving total network observability. In practice, however, the installation process is not implemented at once, but at several stages, because the number of available PMUs at each time period is restricted due to financial problems.

Full system observability will be achieved by installing PMU at each bus, but the PMUs are expensive devices. So it very important to minimize the numbers of PMUs in power system. So different methods are used to compute the minimum required numbers from PMUs in order to fulfill the system observability even PMUs failure or transmission line loss.

An Integer linear programming method had been implemented. The optimal PMUs placement (OPP) will be extended to multi-stages installation. This means that instead of solving OPP in one shot, it will be divided into many stages. New method will be overviewed. That method depends on the decomposition of the power system into sub-networks and computes the optimal PMUs placement (OPP) for each sub-network. 
Because of the power system with considerable size will require a huge numbers of phasor measurement units (PMUs) for full system observability and also due to high cost of PMUs it is very important to find methods for multi-stages of PMUs placement installation.

A new proposed method had been explained. This method is depending on installing a definite number of PMU at each stage. The study case and simulation had been implemented on the IEEE-14 bus system and IEEE-30 bus system.

\section{REFERENCES}

[1] Bei Xu, Ali Abur "Optimal Placement of Phasor Measurement Units for State Estimation" Texas A \& $M$ University. Power Systems Engineering Research Center Publication, Pp.1-14. October 2005.

[2] Bei Gou "Generalized integer linear programming formulation for optimal PMU placement" IEEE Transactions on Power Systems, Volume 23, Issue3, Pp.1099 - 1104, Aug. 2008.

[3] Gomathi Venugopal, Ramachandran. Veilumuth, and Chellammal. Arumugam "Optimal Location of PMUs for complete observability of Power system sub-network" ACEEE International Journal on
Control System and Instrumentation, Vol. 1, No. 1, July 2010.

[4] Ranjana Sodhi, and S.C.Srivastava "Optimal PMUs Placement to Ensure Observability of Power System". Fifteenth National Power Systems Conference (NPSC), IIT Bombay, December 2008.

[5] B.W Kernighan and L. Lin Bell Syst "An Efficient Heuristic Procedure for Partitioning Graphs". Bell System Technical Journal, Volume 49, Issue 2, Pp.291-307, February 1970.

[6] I.O. Habiballah, V.H. Quintana, "Integer-Linear Programming Eigenvector-Based Approach for Multi Partitioning Power System State Estimation Networks" IEEE Proc. on Generation, Transmission \& Distribution. Vol. 141, issue No. 1 , Pp. 11-18, January 1994.

[7] Aaron M. Tenenbaum, "Data Structures Using C". Pearson Education, Prentice Hall, Facsimile edition, Pp.11-18, Dec.1991.

[8] S.B. Yusof, G.J. Rogers, R.T.H. Alden "Slow Coherency Based Network Partitioning Including Load Buses". IEEE Transactions On Power Systems, Vol. 8, Issue No. 3, Pp.1375-1382, August 